\title{
ANÁLISE DO COMPORTAMENTO TEMPORAL DOS PREÇOS DA BORRACHA NATURAL NO MERCADO INTERNACIONAL
}

\author{
TIME SERIES ANALYSIS OF THE BEHAVIOR OF NATURAL RUBBER PRICES IN THE \\ INTERNATIONAL MARKET
}

\author{
Luiz Moreira Coelho Junior ${ }^{1} \quad$ José Luiz Pereira de Rezende $^{2} \quad$ Thelma Sáfadi $^{3} \quad$ Natalino Calegário $^{4}$

\section{RESUMO}

Este trabalho analisou o comportamento dos preços da borracha natural no mercado internacional, no período de janeiro de 1982 a dezembro de 2006, em função de sua oferta e demanda agregadas, evidenciando os principais países produtores e consumidores. Especificamente a pesquisa analisou a evolução dos preços e do quantum comercializado da borracha natural no mercado internacional. Caracterizou, identificou, estimou e analisou modelos para a série de preços reais mensais da borracha crua RSS 1 (US\$/t) e; testou a precisão dos modelos estimados na previsão dos preços dessa commodity, no período de jan./2006 a dez./2006. Os modelos estudados foram das classes ARIMA-ARCH. Os principais resultados encontrados foram: 0s preços reais da borracha natural, no período estudado, apresentam tendência decrescente; A identificação e estimação dos modelos da família ARIMA mostraram a existência de heteroscedasticidade na série estudada e a necessidade de identificar, estimar e analisar os modelos da família ARCH; O modelo que melhor ajustou os retornos da série de preços da borracha crua RSS 1 foi o $\operatorname{AR}(1)$ para um $\operatorname{GARCH}(1,1)$; Os modelos da família ARIMA não satisfizeram as condições de previsão da série estudada; o modelo AR (1)-GARCH $(1,1)$ se mostrou preciso para a realização de prognoses do preço da borracha.

Palavras-chave: economia florestal; borracha natural; séries temporais; modelos ARIMA - GARCH.

\section{ABSTRACT}

This work analyzed the behavior of natural rubber prices in the international market from January 1982 to December 2006 in function of its aggregated demand and supply, pointing out the main producing and consuming countries. Specifically, the research studied the evolution of prices and of the marketed quantum of natural rubber in the international market. It was characterized, identified, estimated and analyzed models for the real monthly prices series of raw rubber RSS 1 (US\$/t), and the accuracy of the estimated models for forecasting prices of this commodity was tested from Jan/2006 to Dez/2006. The studied models were of ARIMA-ARCH class. The main results were: the real natural rubber prices presented decreasing tendency in the period being studied; the ARIMA family estimated model indicating the existence of heteroskedasticity in the series, making it necessary to identify, to estimate and to analyze the models of ARCH family; the model which best adjusted the returns of the price series of the raw rubber RSS1 was AR(1)-GARCH(1,1); the models of the ARIMA family didn't satisfy the prognosis conditions of the series being studied; the AIR (1)-GARCH $(1,1)$ model was accurate for forecasting rubber prices.

Keywords: Forest economy; natural rubber; time series; ARIMA - GARCH models.

\section{INTRODUÇÃO}

O mercado internacional da borracha se compõe de dois grupos - sintética e natural - que possuem comportamentos de consumos diferenciados. Até o final da década de 90, havia concorrência clara entre os volumes consumidos dos dois produtos. No início do século XXI, em razão dos elevados preços do petróleo, o consumo de borracha sintética começou a decrescer. A perda de competitividade da borracha sintética favoreceu o aumento da produção e do consumo da borracha natural.

A Borracha Natural é o produto sólido obtido pela coagulação de látices de determinados vegetais,

1. Economista, M.Sc., Doutorando em Engenharia Florestal pelo Departamento de Ciências Florestais, Universidade Federal de Lavras, Caixa Postal 3037, CEP 37200, Lavras (MG).1mcjunior@hotmail.com

2. Engenheiro Florestal, PhD., Pesquisador visitante do Departamento de Ciências Florestais, Universidade Federal de Lavras, Caixa Postal 3037, CEP 37200, Lavras (MG). jlprezen@ufla.br

3. Licenciada em Matemática, Dr ${ }^{\mathrm{a}}$., Professora Adjunta do Departamento de Ciências Exatas, Universidade Federal de Lavras, Caixa Postal 3037, CEP 37200, Lavras (MG). safadi@ufla.br

4. Engenheiro Florestal, PhD., Professor Adjunto do Departamento de Ciências Florestais, Universidade Federal de Lavras, Caixa Postal 3037, CEP 37200, Lavras (MG). calegari@ufla.br

Recebido para publicação em 3/04/2008 e aceito em 4/05/2009. 
sendo o principal a Hevea Brasiliensis. Essa matéria-prima vegetal proveniente da seringueira, vulgarmente conhecida como borracha já era conhecida dos índios antes do descobrimento da América.

Essa planta é nativa da Amazônia e, embora seja representada por um grande número de espécies que, por uma incisão na casca, exsudam uma secreção leitosa conhecida como látex, somente algumas produzem quantidade e qualidade suficientes para exploração em bases econômicas.

Até o início do século XX, o Brasil detinha o monopólio da produção da borracha natural que contribuiu para alavancar o desenvolvimento do País em seus primórdios. Atualmente, o País não é mais autossuficiente na sua produção. Tailândia, Malásia e Indonésia, hoje, respondem por mais de $80 \%$ da produção mundial da borracha natural, exportando grandes quantidades para os países desenvolvidos (GAMEIRO e SARETTA, 2000).

A borracha natural, que tem como principal substituto a borracha sintética derivada do petróleo, por causa das suas múltiplas aplicações na economia, sobretudo, na indústria automobilística, materiais médicohospitalares, calçados, etc., tornou-se um produto mundialmente valorizado.

Sendo uma "commodity", a borracha tem seus preços estabelecidos pelo mercado internacional, isto é, seus preços não estão atrelados diretamente à oferta doméstica do produto. Sua demanda alta e crescente nas principais economias mundiais, sendo a China seu maior consumidor (PENNACCHIO, 2007).

Os preços dessa commodity variam com as instabilidades macroeconômicas dos países produtores, especialmente por serem economias emergentes. Essas instabilidades afetam os preços do produto no mercado futuro, em especial, Nova York onde é negociada a borracha crua RSS1 que é utilizada para a fabricação de artefatos, como solados, correias e mangueiras (GAMEIRO e SARETTA, 2000).

Os problemas causados por tais variações de preços podem ser previstos e minimizados pelo uso de modelos econométricos, relacionando os fatores causadores com seus efeitos sobre o comportamento do mercado ao longo do tempo. Segundo Wold (1938), as observações presentes e futuras podem ser preditas pela soma da combinação linear de valores passados de uma série de ruídos e um componente determinístico ortogonal.

Tais modelos de prognose de preços são construídos partindo de processos estocásticos especiais que estimam o valor futuro da variável em questão com base somente em seus valores passados (PINDYCK e RUBENFIELD, 1991).

Frequentemente as séries temporais, sobretudo as financeiras e econômicas, apresentam comportamentos incompatíveis com a formulação e previsão por processo linear tais como: mudanças repentinas, variância (condicional) evoluindo no tempo (volatilidade) e irreversibilidade no tempo (MORETTIN e TOLOI, 2006).

A globalização da economia resultou em maior preocupação com relação a risco e incerteza, pois a integração internacional fez com que as atividades produtivas e as estratégias se tornassem muito dinâmicas, envolvendo também questões políticas, econômicas e sociais. Assim, considerar esses processos nas análises dos investimentos é imprescindível para o sucesso empresarial.

Entre as opções para reduzir a incerteza no processo de tomada de decisões econômicas, o uso de modelos de previsão de séries temporais univariadas se destaca como o mais preciso e útil. Esse tipo de análise se aplica nos casos em que há um padrão persistente ou sistemático no comportamento da variável, possível de ser captada através de representação paramétrica (BRESSAN e LIMA, 2002).

Este trabalho analisou o comportamento dos preços da borracha natural no mercado internacional no período de janeiro de 1982 a dezembro de 2006 em função de sua oferta e demanda agregadas, evidenciando os principais países produtores e consumidores.

Especificamente a pesquisa analisou a evolução dos preços e do quantum comercializado da borracha natural no mercado internacional. Caracterizou, identificou, estimou e analisou modelos para a série de preços reais mensais da borracha crua RSS 1 (US\$/t) e; testou a precisão dos modelos estimados na previsão dos preços dessa commodity, no período de jan./2006 a dez./2006. 


\section{MATERIAIS E MÉTODOS}

\section{Objeto de estudo e tratamento dos dados}

A indústria da borracha natural compreende três subsetores: matérias-primas; indústria pesada (pneumáticos) e indústria leve (artefatos de borracha).

No mercado futuro de Nova York, os preços correntes da borracha crua (um tipo de borracha utilizada para fabricação de artefatos, como solados, correias e mangueiras e revestimentos de pisos, etc.), são cotados em dólar americano corrente (i.e. são preços nominais) por tonelada (US\$/t),

Os preços foram coletados do Wall Street Journal (WJS, 2008), no período de janeiro de 1982 a dezembro de 2004, cotados CIF (Cost Insurance and Freight).

A inflação expressa o aumento médio de preços de uma economia ou de segmentos dessa economia, provocando uma perda do poder aquisitivo da moeda. Assim, para analisar a variação de preços ao longo do tempo, é preciso eliminar os efeitos da inflação, isto é, deflacionar a série temporal. Os índices de inflação servem também como indexadores de reajuste dos preços da borracha natural ou de qualquer outro bem ou serviço.

O deflator utilizado foi o Consumer Price Index (CPI) (BLS, 2008). A fórmula para calcular o preço real é dada por:

$$
P_{r}=\frac{P_{n}}{\text { Índice }} * 100
$$

Em que, $P_{r}=$ Preço Real, $P_{n}=$ Preço Nominal ou corrente, Índice = Indicador Econômico.

O período base utilizado para correção dessa série foi dezembro de $2005=100$, para ficar mais próximo da realidade em que os dados foram analisados. Contudo, deve-se evitar usar como base os "anos anormais", isto é, períodos com guerras, recessões e crises econômicas que podem distorcer os valores reais ou deflacionados (HOFFMANN, 2002). A base do deflator escolhida apresenta as características necessárias para ser usada como referência.

Para analisar a série de preços da borracha crua, foram utilizados os retornos compostos instantâneos, dados pela fórmula:

$$
r_{t}=\ln \left(\frac{P_{t}}{P_{t-1}}\right)
$$

Em que, $r_{t}=$ Retorno no tempo $t, P_{t}=$ Preço real da borracha no tempo $t, P_{t-1}=$ Preço real da borracha no tempo $t-1$.

A seguir, serão apresentadas e discutidas as famílias de modelos utilizadas para a determinação e escolha daqueles a serem empregados na pesquisa.

\section{Família ARIMA}

Os modelos ARIMA (Autoregressive, Integrated, Moving Average), introduzidos por Box e Jenkins (1976), baseiam-se na idéia de que uma série temporal não estacionária, do tipo homogêneo, pode ser modelada partindo de $d$ diferenciações e da inclusão de um componente autorregressivo $p$ e de um componente média móvel $q$. Sendo $\left\{Y_{t}\right\}$ um processo que pode ser descrito por meio de uma modelagem $\operatorname{ARIMA}(p, d, q)$ da seguinte forma:

$$
\phi_{p}(B) Y_{t}=\theta_{0}+\theta_{q}(B) a_{t}
$$

Em que, $Z_{t}=\left\{\begin{array}{l}Y_{t}, \text { se o processo é estacionário, quando } d=0 \\ (1-B)^{d} Y_{t}, \text { se o processo não é estacionário, quando } d \geq 1\end{array}\right.$

A ponderação da diferenciação de $Y_{t}$ corresponde a um modelo $\operatorname{ARIMA}(p, d, q)$ com:

$$
\phi_{p}(B)(1-B)^{d} Y_{t}=\theta_{0}+\theta_{q}(B) a_{t}
$$


Em que, $\phi_{p}(B)=1-\phi_{1} B-\phi_{2} B^{2}-\ldots-\phi_{p} B^{p}$ é o operador autorregressivo de ordem $p[A R(p)]$, $\theta_{0}=\mu\left(1-\phi_{1}-\phi_{2}-\ldots-\phi_{p}\right)$ é o intercepto ou a constante, $\theta_{q}(B)=1-\theta_{1} B-\theta_{2} B^{2}-\ldots-\theta_{q} B^{q}$ é o operador de média móvel de ordem $q[M A(q)]$ e $a_{t}$ é um processo de ruído branco (white noise). Se a constante $\theta_{0}$ for diferente de zero, a série integrada proporcionará uma tendência determinística, ou seja, a série apresenta uma tendência crescente ou decrescente, que é independente dos distúrbios aleatórios (PINDYCK e RUBENFIELD, 1991).

A identificação do modelo consiste em determinar sua ordem com base no "princípio de parcimônia". Para isso, foram utilizadas a análise no domínio do tempo (BOX e JENKINS, 1976) e a análise no domínio da frequência (BLOOMFIELD, 1976), sendo os enfoques fundamentais para a análise de séries temporais. Esses enfoques são diferentes e podem ser vistos como distintos, contudo, não são independentes, sendo, na verdade, complementares e ligados matematicamente.

Após a identificação e escolha do modelo apropriado, foram estimados os parâmetros $\phi^{\prime}$ s do processo AR, os parâmetros $\theta$ 's do processo MA. As estimativas dos parâmetros foram feitas pela distribuição gaussiana pelo método dos mínimos quadrados, satisfazendo às condições de invertibilidade $\mathrm{e}$ unicidade dos parâmetros.

Em seguida, para verificar se é ruído branco, foram feitos os diagnósticos do modelo proposto pelas análises dos resíduos padronizados, resíduos da função de Autocorrelação (ACF), resíduos da Função de Autocorrelação Parcial (PACF), do portmanteau test e pelo Critério de Informação de Akaike (AIC) (BOX e PIERCE, 1970; AKAIKE, 1977).

Algumas situações, sobretudo, envolvendo séries econômicas e financeiras, apresentam volatilidade que varia com o tempo. Os modelos lineares da Família $\operatorname{ARIMA}(p, d, q)$ não são ajustados para descrever esse tipo de comportamento, sendo necessário adequar modelos não lineares, como os modelos da Família ARCH (MORETTIN e TOLOI, 2006).

\section{Família ARCH}

Os modelos da família ARCH (Autoregressive Conditional Heteroskedasticity), preconizados por Engle (1982), se relacionam à variância condicional que evolui no tempo. Esses modelos se baseiam na idéia de que o retorno $X_{t}$ é não correlacionado serialmente, mas a variância condicional (volatilidade) é uma função quadrática dos retornos passados.

O modelo ARCH $(r)$ pode ser descrito da seguinte forma:

$X_{t}=\sqrt{h_{t}} \varepsilon_{t}$

$h_{t}=\alpha_{o}+\alpha_{1} X_{t-1}^{2}+\cdots+\alpha_{r} X_{t-r}^{2}$

E que, $\varepsilon_{t} \sim(0,1)$ ou $\varepsilon_{t} \sim t_{v}$ (distribuição $t$ de Student com $v$ graus de liberdade) é uma sequência de variáveis aleatórias independentes identicamente distribuídas (i.i.d.), $\alpha_{0}>0, \alpha_{i}>0, i>0$.

Algumas condições devem ser consideradas para os valores dos coeficientes $\alpha_{i}$, por causa do tipo de imposição que insere no processo $X_{t}$. Uma dessas condições é que a variância condicional dada por $\sigma^{2}$ seja $\operatorname{Var}=\left(X_{t}^{2} \mid F_{t-1}\right)$, sendo, $F_{t-1}=$ ao conjunto de observações até o instante $t$.

O modelo de volatilidade estocástica para uma série $X_{t}$ é:

$$
\begin{aligned}
X_{t} & =\sigma_{t} \varepsilon_{t} \\
\sigma_{t} & =\exp \left(h_{t} / 2\right)
\end{aligned}
$$

Em que $\varepsilon_{t}$ é uma sequência estacionária $(0,1)$ e $h_{t}$ é uma sequência que pode ser estacionária ou não. Contudo, a fórmula mais simples do modelo $\mathrm{ARCH}(s)$ supõe que o logaritmo de volatilidade $h_{t}$ seja 
dado por $h_{t}=\alpha_{0}+\alpha_{1} h_{t-1}+n_{t}$ sendo, $n_{t} \sim N\left(0, \sigma_{n}^{2}\right),\left|\alpha_{1}\right|<1$

A alta persistência na volatilidade das séries de retornos faz com que o valor de $r$ seja elevado no modelo ARCH, ou seja, necessita-se de um grande número de parâmetros de estimados. Bollerslev (1986) propôs o modelo GARCH (Generalized $\mathrm{ARCH}$ ) que é uma generalização do modelo $\mathrm{ARCH}$.

O GARCH expressa alternativamente, de forma mais parcimoniosa, a dependência temporal da variância condicional, no sentido de apresentar menos parâmetros do que o modelo ARCH. O modelo $\operatorname{GARCH}(r, s)$ pode ser descrito da seguinte forma:

$$
\begin{aligned}
& X_{t}=\sqrt{h_{t}} \varepsilon_{t} \\
& h_{t}=\alpha_{o}+\sum_{i=1}^{r} \alpha_{1} X_{t-i}^{2}+\sum_{j=1}^{s} \beta_{1} h_{t-j}
\end{aligned}
$$

Em que $\quad \varepsilon_{t} \quad$ i.i.d. $\quad(0,1) ; \quad \alpha_{0}>0 ; \quad \alpha_{i} \geq 0, i=1, \cdots, r ; \quad \beta_{j} \geq 0, j=1, \cdots, s ; \quad \sum_{j=1}^{q}\left(\alpha_{i}+\beta_{i}\right)<1 ;$ $q=\max (r, s)$.

Para construir um modelo da família ARCH, deve-se primeiro ajustar um modelo ARMA para retirar a correlação serial. Após a construção do modelo ARMA, verifica-se se a série apresenta heteroscedasticidade condicional, por meio do teste de Box-Pierce-Ljung para $X_{t}^{2}$ e o teste de multiplicadores de Lagrange proposto por Engle (1982).

O método da máxima verossimilhança é empregado na maioria das modelagens envolvendo modelos de variância estocástica, tendo características não tendenciosas e consistentes de estimação de parâmetros. Se os distúrbios não apresentarem distribuição normal, pode-se usar o método da quasimáxima verossimilhança.

Para estimar os parâmetros do modelo GARCH, utilizou-se o método da máxima verossimilhança condicional. Mesmo que a distribuição não seja gaussiana, segundo Bollerslev e Wooldridge (1992) se pode assumir que os distúrbios sejam gaussianos.

Para a sequência $\tilde{X}_{t}$, a verificação da adequação do modelo proposto é feita pela estatística $Q$ de Ljung-Box. Os resíduos do modelo são variáveis aleatórias i.i.d., verificado pelo Teste $t$-Student ou distribuição normal-padrão e avaliado por meio do Critério de Informação de Akaike (AIC).

A prognose de $t$ passos à frente, com origem em $t$, é dada por

$\hat{h}_{t}(\imath)=\alpha_{0}+\sum_{i=1}^{r} \alpha_{i} \hat{h}_{t}(l-i)+\sum_{j=1}^{s} \beta_{j} \hat{h}_{t}(l-j)$

Em que $\hat{h}_{t}(l-i)=X_{t+l-i}^{2}$ se $\imath-i \leq 0$ e $\hat{h}_{t}(l-j)=X_{t+l-i}^{2}$ se $l-j \leq 0$.

Os dados foram processados pelos softwares Excel, SAS e S-Plus 2000.

\section{RESULTADOS E DISCUSSÃO}

A Tabela 1 mostra a evolução da produção e do consumo da borracha natural, no período de 1984 a 2005, indicando que os principais países produtores são Tailândia, Indonésia e Malásia e os principais consumidores China, Estados Unidos e Japão.

Nos anos 80, Malásia e Indonésia produziam em média $40 \%$ do total e lideravam o ranking da produção mundial da borracha natural. A partir da década de 90 , começaram a perder a primeira posição para a Tailândia. Do lado do consumo, também houve modificação com a China ultrapassando os Estados Unidos e se tornando o maior consumidor, atingindo 1,826 milhões de toneladas, ou seja, $20,8 \%$ do consumo mundial, em 2005.

De acordo com a Teoria do Equilíbrio Parcial de Marshall, os preços são determinados pelo mercado internacional (oferta e demanda). Do lado da oferta, os exportadores acrescentam ao custo de produção o mark up. Do lado da demanda, os países consumidores tentam minimizar os custos de importação. Do jogo dessas duas forças e da influência do fator tempo chega-se ao preço de equilíbrio. 
TABELA 1: Evolução do balanço mundial de borracha natural $-1.000 \mathrm{t}$ (peso seco).

TABLE 1: Evolution of the world balance of natural rubber - $1.000 \mathrm{t}$ (dry weight).

\begin{tabular}{lcccccccccc}
\hline Países & 1984 & 1987 & 1990 & 1993 & 1996 & 1999 & 2002 & 2005 \\
\hline Produção & 4255 & 4850 & 5120 & 5300 & 6330 & 6820 & 7303 & 8703 \\
\hline Tailândia & 629 & 926 & 1275 & 1553 & 1970 & 2346 & 2615 & 2833 \\
Indonésia & 116 & 1203 & 1262 & 1301 & 1543 & 1501 & 1630 & 2271 \\
Malásia & 1531 & 1579 & 1291 & 1074 & 1083 & 928 & 890 & 1126 \\
Índia & 184 & 227 & 324 & 428 & 540 & 629 & 641 & 772 \\
Vietnã & 189 & 238 & 264 & 326 & 430 & 445 & 468 & 428 \\
China & 142 & 122 & 113 & 104 & 113 & 97 & 91 & 104 \\
Outros & 464 & 555 & 591 & 514 & 651 & 874 & 968 & 1169 \\
\hline Consumo & 4230 & 4800 & 5210 & 5440 & 6130 & 6630 & 7542 & 8777 \\
\hline China & 402 & 545 & 600 & 650 & 810 & 852 & 1310 & 1826 \\
EUA & 751 & 789 & 808 & 967 & 1002 & 1116 & 1111 & 1159 \\
Japão & 525 & 568 & 677 & 631 & 558 & 734 & 749 & 857 \\
Índia & 213 & 278 & 358 & 444 & 473 & 619 & 680 & 789 \\
Malásia & 190 & 199 & 209 & 175 & 193 & 226 & 247 & 263 \\
Tailândia & 162 & 170 & 179 & 169 & 182 & 240 & 231 & 230 \\
Outros & 1987 & 2251 & 2379 & 2404 & 2912 & 2843 & 3214 & 3653 \\
\hline
\end{tabular}

Fonte: AGRIANUAL (2008).

Mesmo as moedas fortes (dólar norte americano e libra esterlina) frequentemente usadas no mercado internacional sofrem os efeitos inflacionários. Assim, para que se possa compará-las, é preciso corrigir ou eliminar tais efeitos.

A Figura 1 mostra o comportamento dos preços reais e nominais da borracha crua RSS 1 (USS/t) CIF praticados no mercado spot de Nova York. Observa-se que houve desvalorização da moeda ao longo do tempo.

Nota-se que as variações longitudinais das séries de preços da borracha crua RSS 1 estão decrescendo ao longo do tempo. Para identificar os modelos apropriados, inicialmente, devem ser analisados os gráficos originais das séries em estudo (Figura 1). A análise gráfica indicou a presença de tendência e alteração na variância, diante disso, realizaram-se alguns testes para determinar a série é ou não estacionária.

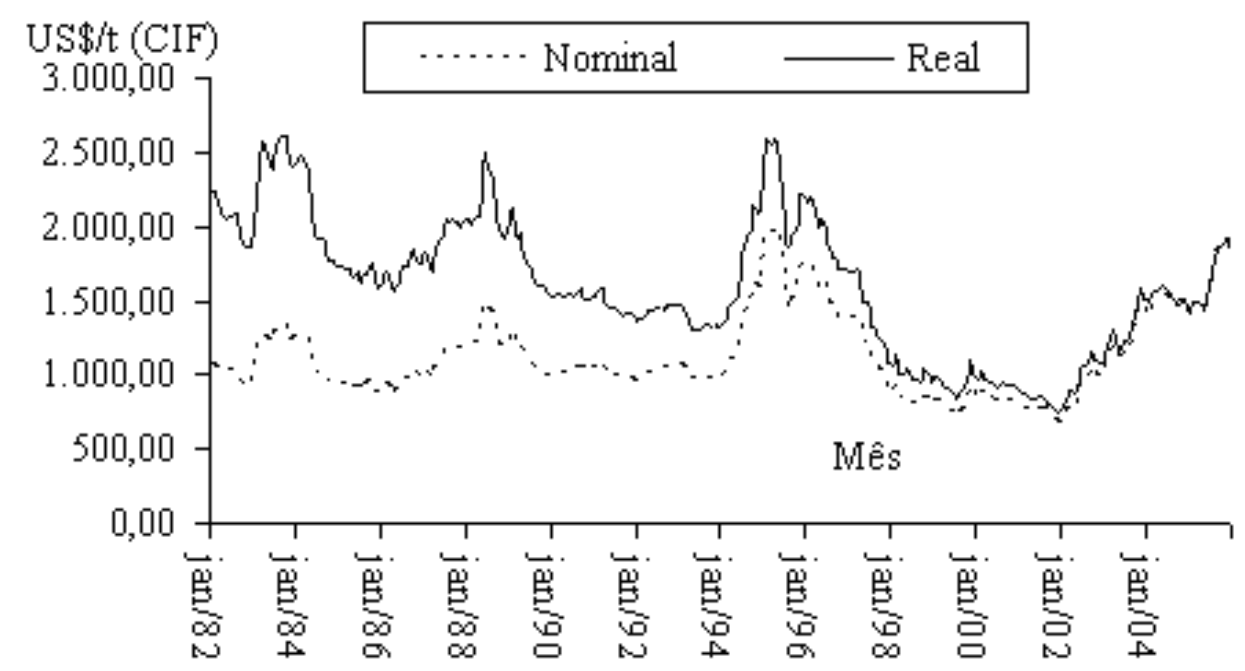

FIGURA 1: Comportamento dos preços reais e nominais da Borracha Natural (RSS 1), no mercado spot em Nova York, no período de Jan/1982 a Dez/2005 (Base Dez/2005 = 100).

FIGURE 1: Behavior of real and nominal prices of the Natural Rubber (RSS 1), in the New York spot market, from Jan/1982 to Dez/2005 (Base Dez/2005 = 100). 
O teste de Cox-Stuart detectou a existência de tendência e que a série original é não estacionária, confirmando a análise gráfica. Assim, para identificar o modelo apropriado, utilizaram-se os retornos da série de preços da borracha crua RSS 1 (Figura 2) e foram geradas a Função de Autocorrelação (ACF) e Função de Autocorrelação Parcial (PACF).

Nota-se que a ACF apresenta uma mistura de funções senoidais e exponenciais amortecidas, típico de um processo auto-regressivo (AR). A PACF determina a ordem $p$ do auto-regressivo $\operatorname{AR}(p)$, em que apresenta uma defasagem (lag 1) significativa, fornecendo indícios de um AR (1), ou seja, $\left(1-\phi_{1} B\right) Z_{t}=a_{t}$.

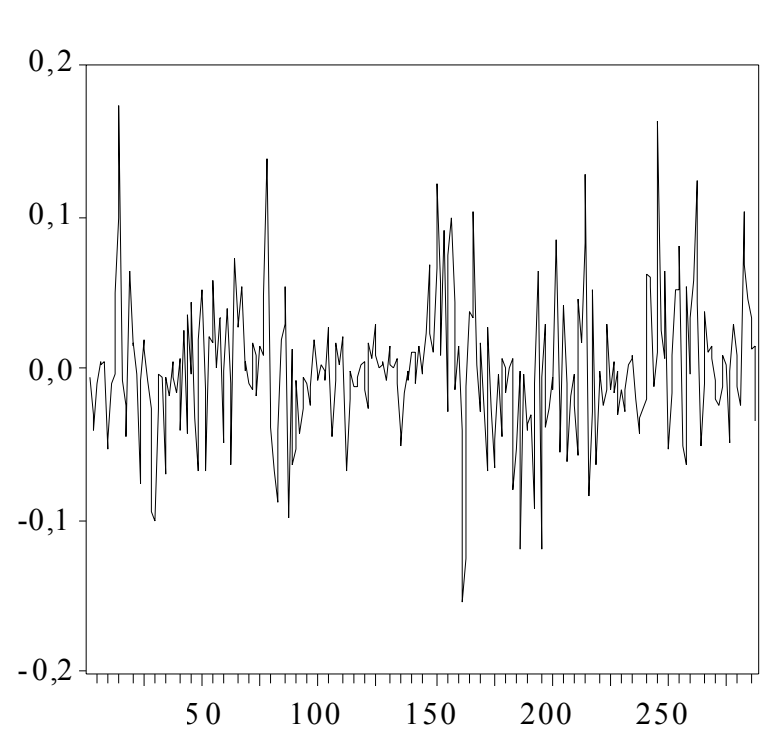

\begin{tabular}{|c|c|}
\hline$\triangle C F$ & PACF \\
\hline 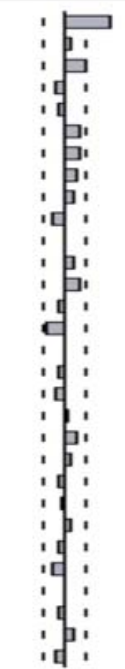 & 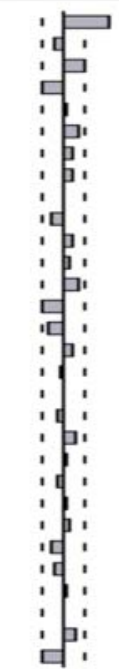 \\
\hline
\end{tabular}

FIGURA 2: Retornos mensais, Função de Autocorrelação (ACF) e Função de Autocorrelação Parcial (PACF) do retorno da borracha natural no mercado spot de Nova York.

FIGURE 2: Monthly economic return, Autocorrelation Function (ACF) and Partial Autocorrelation Function (PACF) of the return of the natural rubber in the New York spot market.

Foram testados vários modelos do tipo ARMA (p, q), para captar os efeitos temporais na série. Os parâmetros foram estimados pelo método dos mínimos quadrados. O modelo autorregressivo de ordem $p=1$, ou seja, [AR (1)] se ajustou melhor, de acordo com os critérios apresentados na Tabela 2.

TABELA 2: Estimativa dos parâmetros do modelo AR (1).

TABLE 2: Parameter estimates of the AR (1) model.

\begin{tabular}{l|c|c|c|c}
\hline & Coeficientes & Erro-padrão & Estatística $\mathrm{z}$ & p-Valores \\
\hline$\phi_{1}$ & 0,266774 & 0,057150 & 4667984 & 0,0000 \\
\hline $\mathrm{R}^{2}$ & & \multicolumn{3}{|c}{0} \\
$\mathrm{AIC}$ & & \multicolumn{3}{|c}{$-3,4136833$} \\
$\mathrm{SBIC}$ & & $-3,400899$ & \\
\hline
\end{tabular}

A equação do modelo AR(1) estimada assume a seguinte forma:

$(1-0,2668 B) Y_{t}=a_{t}$

O "portmanteu test" utilizado e sugerido por Box \& Jenkins (1976) verifica se há ruído branco ou não, por meio da estatística "Q", fazendo ainda as análises gráficas dos resíduos padronizados das ACF e PACF. A Figura 3.a mostra as ACF e PACF dos resíduos do modelo AR (1) indicando que é ruído branco.

Contudo, as análises das ACF e PACF dos resíduos quadráticos da série defasada da base (Figura 3.b) indicaram que há necessidade de verificar a heteroscedasticidade na variância dos resíduos da série modelada com o AR (1).

O teste do Multiplicador de Lagrange, proposto por Engle (1982), do tipo - ARCH LM test -, 
evidenciou a existência de heteroscedasticidade, conforme Tabela 3. Com base no padrão heterocedástico da volatilidade para a média condicional dos resíduos, utiliza-se o método da família ARCH para modelar a série de retorno da borracha.

TABELA 3: Teste heteroscedasticidade ARCH-LM.

TABELA 3: ARCH-LM heteroscedasticity test.

\begin{tabular}{l|c|c|c}
\hline Estatística-F & 5,125124 & Probabilidade & 0,024339 \\
\hline Obs*R ${ }^{2}$ & 5,069535 & Probabilidade & 0,024350 \\
\hline
\end{tabular}

Os parâmetros do modelo foram estimados pelo método do Multiplicador de Lagrange, e os resultados da estimação para a média condicional e para a volatilidade da série de retorno da borracha são apresentados na Tabela 4.

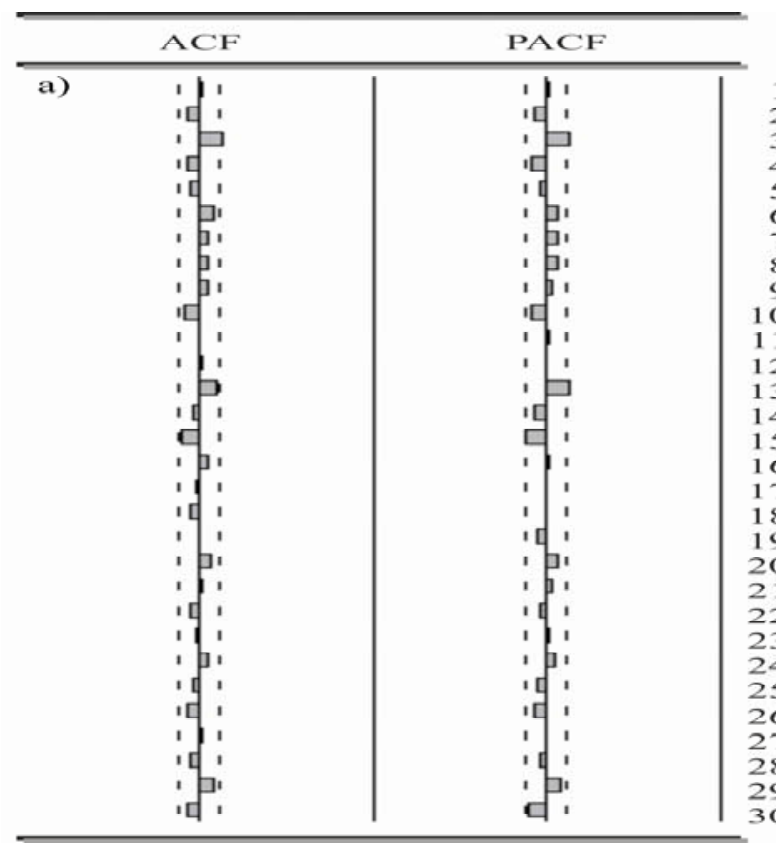

\begin{tabular}{|c|c|c|c|}
\hline \multicolumn{2}{|r|}{$\triangle C F$} & \multicolumn{2}{|c|}{ PACF } \\
\hline b) & 口 & 口 & 1 \\
\hline b) & 10 & if 1 & 2 \\
\hline & ין & ים & 3 \\
\hline & ין & i i & 4 \\
\hline & , 户 & ין" & 5 \\
\hline & 1 & 10 & 6 \\
\hline & 11 & 11 & 7 \\
\hline & ין & ין י & 8 \\
\hline & 1. & , [1 & 9 \\
\hline & 11 & \begin{tabular}{l|l}
1 & 1
\end{tabular} & 10 \\
\hline & ין י & י & 11 \\
\hline & 1,1 & 1 $[1$ & 12 \\
\hline & . & . & 13 \\
\hline & . 1 & 17 & 14 \\
\hline & 11 & 1 & 15 \\
\hline & 11 & 1 & 16 \\
\hline & 1 & 1 & 17 \\
\hline & 1 & 18 & 18 \\
\hline & 1 & 14. & 19 \\
\hline & ' & ' & 20 \\
\hline & . & 1. & 21 \\
\hline & is. & 10 & 22 \\
\hline & . & 11 & 23 \\
\hline & 18, & 10 & 24 \\
\hline & 11 & 111 & 25 \\
\hline & & ' & 26 \\
\hline & 17 & י' & 27 \\
\hline & 11 & 1 1 & 28 \\
\hline & . & in: & 29 \\
\hline & ' 口 & ' 口 & 30 \\
\hline
\end{tabular}

FIGURA 3: Função de autocorrelação (ACF) e Função de Autocorrelação Parcial (PACF) dos resíduos do AR (1) e ACF e PAC dos quadrados dos resíduos do retorno da borracha natural no mercado spot, em Nova York.

FIGURE 3: Autocorrelation function (ACF) and Partial Autocorrelation Function (PACF) of the residues of the AtR (1) and ACF and PACF of the squares of the residues of the return of the natural rubber in the New York spot market.

TABELA 4: Ajustamento modelo AR (1) - GARCH $(1,1)$.

TABLE 4: AR (1) - GARCH $(1,1)$ model adjustment.

\begin{tabular}{lcccc}
\hline & Coeficientes & Erro-padrão & Estatística $\mathrm{z}$ & p-Valores \\
\hline$\phi_{1}$ & 0,276580 & 0,066131 & 0,0000 \\
\hline$\alpha_{0}$ & \multicolumn{3}{c}{ Equação da Variância } \\
$\alpha_{1}$ & $9,32 \mathrm{E}-05$ & 2,889175 & 0,0039 \\
$\beta_{1}$ & 0,000269 & 0,065322 & 3,403670 & 0,0007 \\
\hline AIC & 0,222333 & 0,078485 & 8,473366 & 0,0000 \\
SBIC & 0,665030 & & $-3,455701$ & \\
Soma dos quadrados dos resíduos & & $-3,404568$ & \\
\hline
\end{tabular}

O modelo, que melhor ajustou a série, foi o $\operatorname{AR}(1)$ para um $\operatorname{GARCH}(1,1)$ corrigido pelos resíduos do modelo AR(1) para a média condicional dos retornos e confirmados pelos critérios de Informação de Akaike 
(AIC), Schwarz (SBIC) e a Soma dos Quadrados dos Resíduos.

A equação do modelo $\operatorname{AR}(1)-\operatorname{GARCH}(1,1)$ assume a seguinte forma:

$$
\begin{aligned}
& Y_{t}=\phi_{1} Y_{t-1}+X_{t}, \quad X_{t}=\sqrt{h_{t}} \varepsilon_{t} \\
& h_{t}=\alpha_{0}+\alpha_{1} X_{t-1}^{2}+\beta_{1} h_{t-1}
\end{aligned}
$$

substituído os parâmetros por seus valores, tem-se:

$$
\begin{aligned}
& Y_{t}=0,2766 Y_{t-1}+X_{t}, \quad X_{t}=\sqrt{h_{t}} \varepsilon_{t} \\
& h_{t}=0,0003+0,2223 X_{t-1}^{2}+0,6650 h_{t-1}
\end{aligned}
$$

Os resultados da estatística de Box-Ljung dos resíduos quadráticos indicam que a heterocedasticidade da variância dos resíduos foi controlada (Figura 4).

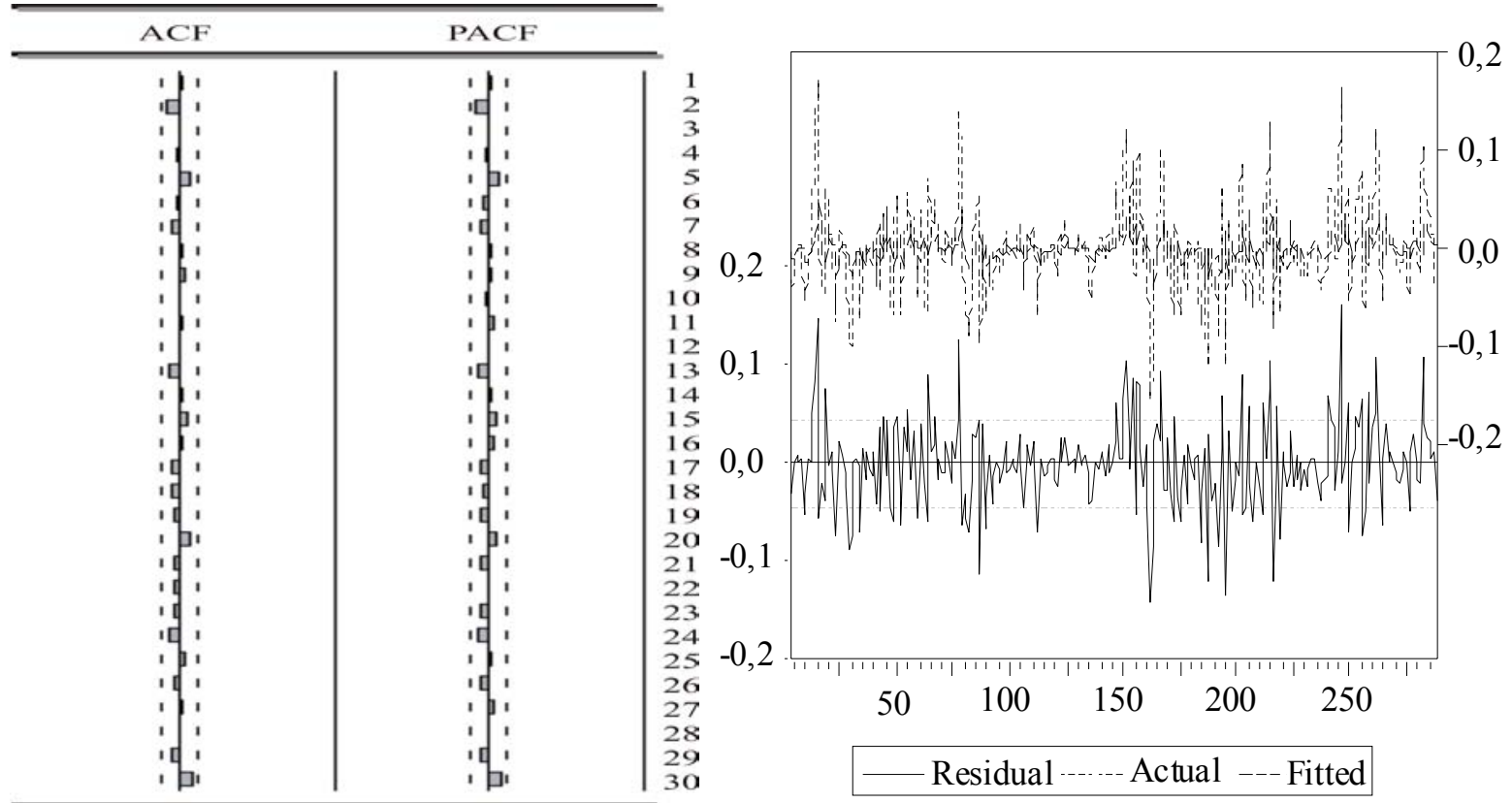

FIGURA 4: Função de autocorrelação (ACF) e Função de Autocorrelação Parcial (PACF) dos resíduos do $\operatorname{GARCH}(1,1)$ e gráfico do retorno ajustado e real.

FIGURE 4: Autocorrelation function (ACF) and Partial Autocorrelation Function (PACF) of the residues of the $\operatorname{GARCH}(1,1)$ and real and return graphic.

O teste Multiplicador de Lagrange revela que o modelo está bem ajustado (Tabela 5).

A estimativa dos retornos dos preços realizada pelo modelo AR (1) - GARCH $(1,1)$ com intervalo de confiança de $95 \%$ para os limites superiores e inferiores da série é apresentada na Figura 5.

TABELA 5: Teste de hetoroscedasticidade ARCH-LM.

TABLE 5: ARCH-LM heteroscedasticity test.

\begin{tabular}{l|l|l|l}
\hline Estatística-F & 0,031672 & Probabilidade & 0,858877 \\
\hline Obs. $\mathrm{R}^{2}$ & 0,031892 & Probabilidade & 0,858265 \\
\hline
\end{tabular}



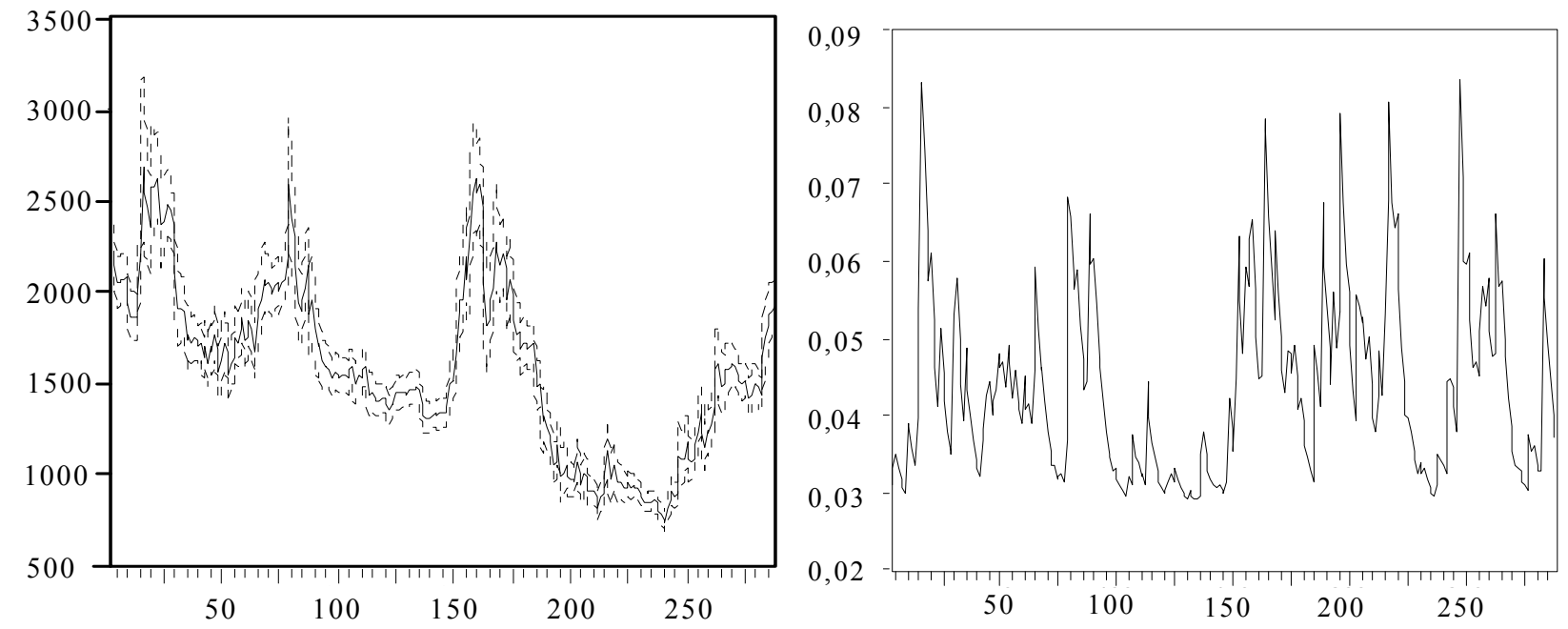

FIGURA 5: Estimativa e volatilidade da série Modelo AR (1) - GARCH $(1,1)$.

FIGURE 5: Estimate and volatility of AR(1) - GARCH $(1,1)$ model.

Após o ajuste do modelo da série de retornos da borracha crua RSS1, no período de janeiro de 1982 a dezembro de 2005, para testar a precisão do modelo de previsão, utilizou-se uma amostra correspondente aos 7 meses seguintes (janeiro a julho de 2006). A Figura 6 apresenta a prognose para série de preços da borracha crua RSS 1.

O modelo ajustado produziu boa prognose dos preços futuros, minimizando os erros entre os valores reais e os previstos. O erro quadrático médio da previsão é igual a $33.524,73$, sendo a maior variação entre o valor observado e as projeções de $9,94 \%$ em abril.

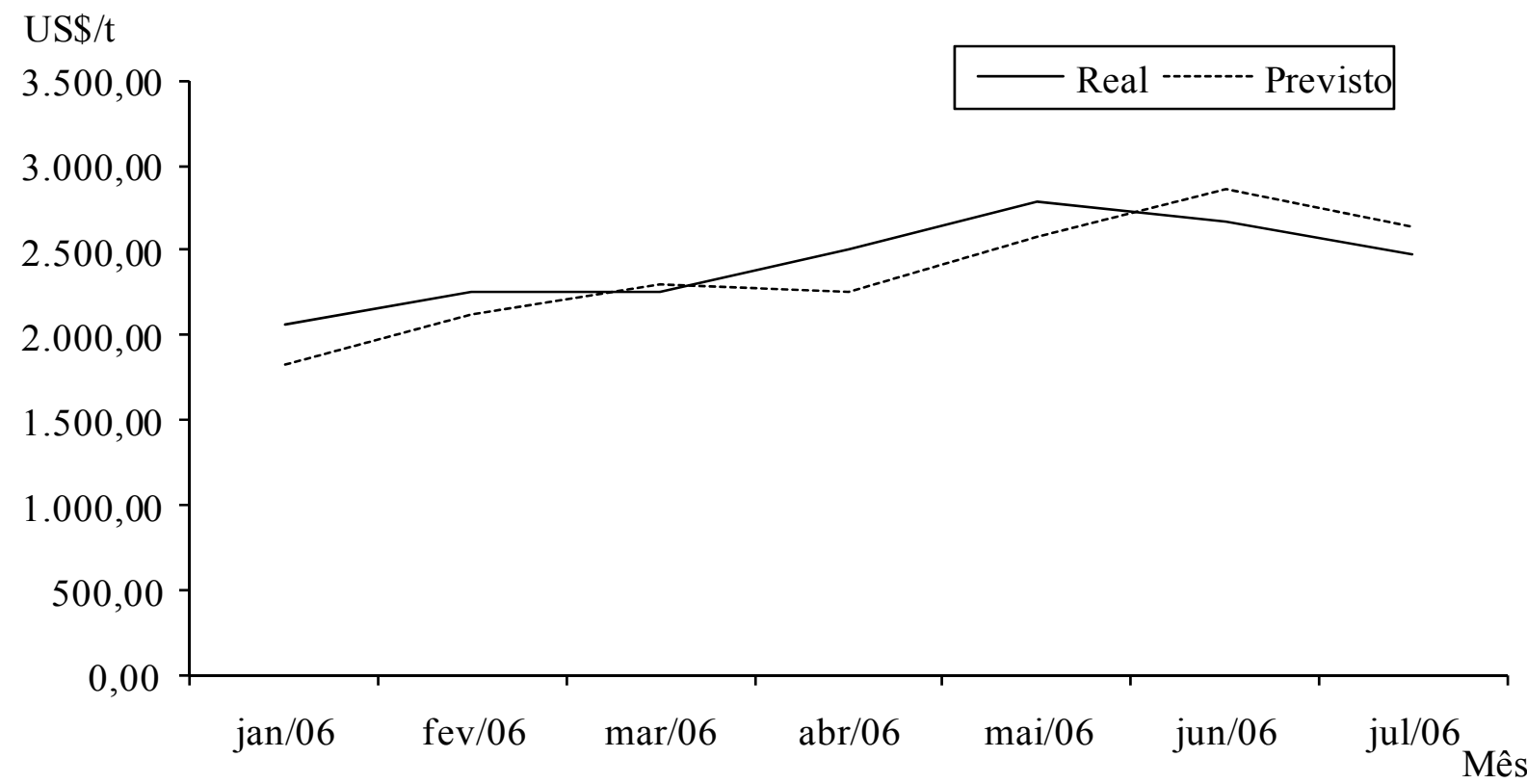

FIGURA 6: Gráfico da previsão dos preços da borracha crua RSS 1 (US\$/t) (linha tracejada) e dos preços reais observados (linha cheia).

FIGURE 6: Graph of the forecast real price series (dotted line) and observed real prices (full line) of raw rubber RSS 1 (US\$/t). 


\section{CONCLUSÃO}

Partindo-se das análises feitas neste trabalho, chegou-se às seguintes conclusões.

Os preços reais da borracha natural, no período estudado, apresentaram tendência decrescente;

A identificação, estimação e validação dos modelos da família ARIMA mostraram a existência de heteroscedasticidade na série estudada e a necessidade de identificar, estimar e analisar os modelos da família $\mathrm{ARCH}$;

O modelo que melhor ajustou os retornos da série de preços da borracha crua RSS 1 foi o AR(1) para um $\operatorname{GARCH}(1,1)$;

Os modelos da família ARIMA não satisfizeram as condições de previsão da série estudada;

A prognose dos valores "futuros" realizada pelo modelo AR (1)-GARCH (1,1), para o ano de 2005, se mostrou precisa.

\section{REFERÊNCIAS BIBLIOGRÁFICAS}

AGRIANUAL. Anuário da agricultura brasileira. São Paulo: FNP, 1996 a 2008.

AKAIKE, H. On entropy maximization principle. In: KRISHAIAH, P. R. (Ed.). Application of statistics. Amsterdam, The Netherlands: North-Holland, 1977. p. 27-41.

BLOOMFIELD, P. Fourier analysis of times series: an introduction. New York: John Wiley, 1976.

BLS. BUREAU OF LABOR STATISTIC. Consumer price index. Washington: U. S. Department of Labor, Disponível em: $<\mathrm{ftp}: / / \mathrm{ftp} . b l s . g o v /$ pub/special.request/cpi/cpiai.txt $>$. Acesso em: 15 out. 2008.

BOLLERSLEV, T. Generalized autoregressive conditional heteroskedasticity. Journal of Econometrics, v. 31, n. 3, p. 307-327, Apr. 1986.

BOLLERSLEV, T; WOOLDRIDGE, J. M. "Quasi-maximun likelihood estimation and inference in dynamic models with time-VaRying CoVaRiances". Econometric Reviews, v. 11, n. 2, p. 143-172, Feb./Mar. 1992.

BOX, G. E. P.; JENKINS, G. M. Time series analysis: forecasting and control. San Francisco: Holden-Day, 1976.

BOX. G. E. P.; PIERCE, D. A. Distribution of residuals autocorrelations in autoregressive-integrated moving average time series models. Journal of the American Statistical Association, Washington, v. 65, n. 332, p. 1509-1526, Dec. 1970.

BRESSAN, A. A.; LIMA, J. E. Modelos de previsão de preços aplicados aos contratos futuros de boi gordo na BM\&F. Nova Economia, Belo Horizonte, v. 12, n. 1, p. 117-140. jan./jun. 2002.

ENGLE, R. F. Autoregressive conditional heteroskedasticity with estimates of the variance of U. K. inflation. Econometrica, Princeton, v. 50. n. 4, p. 987-1008. Oct./Dec.1982.

GAMEIRO, A. H.; SARETTA, C. B. Preços do petróleo puxam os da borracha. Revista Preços Agrícolas, São Paulo, n. 168 , p. 29 , out./nov. 2000 .

HOFFMANN, R. Estatística para Economistas. 3. ed. rev. e ampl. São Paulo: Pioneira Thomson Learning, 2002, $430 \mathrm{p}$

MORETTIN, P. A.; TOLOI, C. M. C. Análise de séries temporais. 2. ed. ver. e ampl. São Paulo: E. Blücher, 2006.

PENNACCHIO, H. L. Conjuntura semanal: borracha. CONAB. Disponível em $<$ www.conab.gov.br $>$ acesso em 20/11/2007.

PINDYCK, R. S.; RUBENFIELD, D. L. Econometric models and economic forecasts. 3rd ed., New York: McGrawHill, 1991

WOLD, H. O. A study in the analysis of stationary time series. Sweden: Uppsala, 1938. $214 \mathrm{p}$.

WSJ. Wall Street Journal. Disponivel em: $<$ http://online.wsj.com $/ \mathrm{mdc} /$ page/marketsdata.html $>$ acesso em: 20 de set. de 2007 